\title{
USO DE MODELOS DE FILAS PARA REORGANIZAÇÃO DO PROCESSO DE ATENDIMENTO A CLIENTES DE UM TERMINAL DE DISTRIBUIÇÃO DE COMBUSTÍVEIS: UM ESTUDO DE CASO
}

\author{
Sandra Pelufe \\ Universidade Federal do Rio Grande \\ Av. Itália, s/n - Km 8 - Carreiros, Rio Grande - RS \\ sandra_pelufe@hotmail.com \\ Diogo Garcia Storino \\ Universidade Federal do Rio Grande \\ Av. Itália, s/n - Km 8 - Carreiros, Rio Grande - RS \\ diogo.storino@hotmail.com \\ André Andrade Longaray \\ Universidade Federal do Rio Grande \\ Av. Itália, s/n - Km 8 - Carreiros, Rio Grande - RS \\ andrelongaray@gmail.com \\ Paulo Roberto da Silva Munhoz \\ Universidade Federal do Rio Grande \\ Av. Itália, s/n - Km 8 - Carreiros, Rio Grande - RS \\ paulorsmunhoz@gmail.com \\ Thauane Adamoli Amaral \\ Universidade Federal do Rio Grande \\ Av. Itália, s/n - Km 8 - Carreiros, Rio Grande - RS \\ thauaneadamoli@gmail.com
}

\begin{abstract}
RESUMO
Este artigo tem por objetivo a utilização de um Modelo de Simulação baseado na Teoria das Filas visando a melhoria do atendimento a clientes de um Terminal de Distribuição de Combustíveis. Em relação a metodologia, a pesquisa classifica-se como predominantemente qualitativa, pois as informações dos trabalhos analisados possuem caráter exploratório-interpretativo. Quanto ao delineamento, a pesquisa caracteriza-se como um estudo de caso. Para tal, foram adotadas a observação participante de forma aberta e a consulta à documentos, na forma de relatórios mensais em uma empresa atuante no Setor. Entre os resultados obtidos através das simulações, é possível observar que, mesmo com o aumento significativo do número de caminhões atendidos pela empresa, na ocorrência de uma pequena elevação da capacidade de atendimento, observa-se uma redução no tempo de permanência dos caminhões na fila.
\end{abstract}

Palavra-chave: Teoria das Filas; Simulação; Distribuição; Combustíveis. 


\begin{abstract}
This paper aims to use a Simulation Model based on Queue Theory to improve customer service at a Fuel Distribution Terminal. Regarding the methodology, the research is classified as predominantly qualitative, because the information of the analyzed works has exploratory-interpretative character. As for the design, the research is characterized as a case study. To this end, open participant observation and consultation of documents were adopted, in the form of monthly reports in a company operating in the Sector. Among the results obtained through the simulations, it is possible to observe that, even with the significant increase in the number of trucks served by the company, in the event of a small increase in the service capacity, there is a reduction in the time spent by trucks in the queue.
\end{abstract}

Keywords: Queue Theory; Simulation; Distribution; Fuel.

\title{
Como Citar:
}

PELOFE, Sandra; GARCIA, Diogo Garcia; LONGARAY, André Andrade; MUNHOZ, Paulo Roberto da Silva; AMARAL, Thauane Adamoli. Uso de modelos de filas para reorganização do processo de atendimento a clientes de um terminal de distribuição de combustíveis: um estudo de caso. In: SIMPÓSIO DE PESQUISA OPERACIONAL E LOGÍSTICA DA MARINHA, 19., 2019, Rio de Janeiro, RJ. Anais [...]. Rio de Janeiro: Centro de Análises de Sistemas Navais, 2019.

\section{INTRODUÇÃO}

No final da década de noventa ocorreu à flexibilização do monopólio do petróleo, permitindo assim, que atividades antes sob o poder da União pudessem ser efetuadas por outras empresas além da empresa Petróleo Brasileiras S. A. - Petrobras. Esta flexibilização iniciou-se a partir da regulamentação através da Lei do Petróleo ( $\mathrm{N}^{\circ}$ 9.478, de 06.08.97) que discorre sobre a política energética nacional, atividades relacionadas ao monopólio do petróleo, além de instituir o Conselho Nacional de Política Energética e a Agência Nacional do Petróleo, além de outras providências. Frente ao exposto, independente da origem de seu capital, qualquer empresa pode executar as atividades de exploração, produção, transporte, refino, importação e exportação de petróleo. No ano de 2002, a abertura do mercado se estendeu ao setor downstream, possibilitando que as distribuidoras possam desfrutar dessa facilidade na importação dos derivados de petróleo [13].

A cadeia de abastecimento da indústria do petróleo está dividida em dois segmentos principais, as quais são upstream e downstream. A cadeia de abastecimento upstream envolve a aquisição de petróleo bruto, que é a especialidade das companhias de petróleo, incluindo a exploração, previsão, produção e gerenciamento logístico de fornecimento de petróleo bruto de poços de petróleo localizados remotamente para refinarias. A cadeia de abastecimento downstream inicia-se na refinaria, onde o petróleo bruto é transformado nos produtos consumíveis que são a especialidade de refinarias e petroquímicas. Envolve o processo de previsão, produção e gerenciamento logístico de entrega de derivados de petróleo bruto a clientes em todo o mundo [6].

Com relação às operações logísticas, esta regulamentação permitiu ampliar as perspectivas econômicas e sociais para todos os segmentos relacionados direta ou indiretamente com os mercados de óleo, derivados e gás natural. No setor de downstream da indústria, a abertura do mercado possibilitou uma reestruturação na área logística, com a inclinação da integração ao mercado global, onde os produtos podem ser adquiridos de 
fornecedores de qualquer parte do continente, contratação de transporte internacional e distribuição no mercado interno para o consumidor final [2].

Há no mercado brasileiro uma demanda crescente por combustíveis, o qual está ligado diretamente à dificuldade em expandir a infraestrutura, existindo também uma preocupação relacionada ao abastecimento desses produtos. O país conta atualmente com dezessete refinarias de petróleo, espalhadas por nove estados, localizadas em sua maioria na região sudeste [8]. Duas são de propriedade particular e as demais pertencem à Petrobrás. Em 2016 foram comercializados $135.441 .654 \mathrm{~m}^{3}$ de derivados de petróleo [1].

Os combustíveis líquidos podem ser transportados por praticamente todos os modais, com exceção do aéreo, através de dutos, ferrovias, hidrovias, cabotagem ou rodovias. Por falta de modais competitivos, a maior parte do abastecimento de combustíveis é feita por transporte terrestre, através de caminhões. Além das dificuldades em infraestrutura, as regulamentações do setor também dificultam a realização de investimentos, principalmente nas questões ligadas aos licenciamentos ambientais, uma vez que a atividade de distribuição dos combustíveis implica em um elevado risco [9].

O Instituto Brasileiro de Petróleo, Gás e Biocombustíveis (IBP) oferece uma contribuição para o desenvolvimento dos segmentos de refino e petroquímica, GLP, asfalto, combustíveis e biocombustíveis, lubrificantes, laboratório, logística de distribuição de combustíveis, portos e terminais, e transporte dutoviário, através do trabalho desenvolvido por suas comissões técnicas atuantes em cada um dos segmentos. Essas áreas constituem o segmento downstream, abrangendo o processamento do petróleo bruto e o escoamento dos derivados utilizados pela indústria, comércio e consumidores finais. O Instituto atua também na melhoria da infraestrutura de portos e na perspectiva do transporte por dutos de derivados de petróleo [7].

Ainda de acordo o IBP, atualmente no Brasil, 90\% da movimentação dos derivados do petróleo é realizada por caminhões, através das rodovias, devido a uma insuficiência de ferrovias, dutos e hidrovias. Como resultado, há um aumento nos custos em fretes, no consumo de óleo diesel e dos riscos de acidentes. A IBP (2018) calcula que, apenas no setor portuário, seriam necessários investimentos de R\$ 3,1 bilhões em dez anos para superar os bloqueios à movimentação de gasolina, diesel, querosene de aviação e etanol.

No Brasil há grande disponibilidade de petróleo para exploração, porém a distribuição de $80 \%$ do volume está concentrada em quatro companhias. Elas possuem bases de armazenamento, assim como um sistema de logística robusto para suprir o atendimento do mercado. Cada empresa possui uma propriedade integral ou compartilhada de terminais de armazenagem em todo o território nacional. Os terminais estão estrategicamente localizados próximos a refinarias e usinas e a clientes finais, com propósito de atender as demandas com um nível de serviço adequado, qualidade e baixo custo. As empresas distribuidoras são responsáveis por coordenar os fluxos de recebimento e entrega de combustíveis de forma a garantir o abastecimento de todo o país, acompanhando o planejamento de vendas adotado por cada uma. Os produtos comercializados pelas distribuidoras são provenientes de refinarias e usinas, os quais são basicamente gasolina, diesel, biodiesel, gás natural e etanol [11].

Um dos grandes desafios enfrentados pelas distribuidoras de combustíveis é possuir bases de armazenamento capazes de atender à toda demanda com eficiência e segurança. Dessa forma são necessários investimentos em equipamentos, novas tancagens, melhorias de processos e segurança, porém não na velocidade que deveriam ocorrer, uma vez que alterações físicas resultam em altos custos financeiros com retorno à longo prazo. Na tentativa de tornar os processos em uma base distribuidora mais eficiente, são realizados constantemente estudos e análises, principalmente quando a operação não é capaz de atender um aumento de demanda e a formação dos gargalos logísticos, como as filas de caminhões 
que aguardam para realizar o carregamento nos terminais [11].

Em busca de melhorar o atendimento aos clientes de uma distribuidora de combustíveis, o trabalho tem como propósito o desenvolvimento de um modelo de simulação, baseado na Teoria das Filas, adotando para tal apenas o modal de transporte rodoviário.

\section{REFERENCIAL TEÓRICO}

As filas não são agradáveis e dependendo da espera, trazem prejuízos e aborrecimentos. Além disso, apresentam o lado desfavorável de aumentar os custos. No entanto, necessita-se conviver com as mesmas, visto que se torna economicamente inviável superdimensionar um sistema para que nunca ocorram filas. Dessa forma, tenta-se construir um balanceamento adequado, permitindo um atendimento aceitável e com custo reduzido [12].

Sistemas de fila são considerados como uma linha de clientes que aguardam a prestação de serviços de um ou mais prestadores [4]. Uma vez que a demanda ultrapassa a capacidade existente do servidor a formação dessas filas ocorre.

Teoria das Filas e Simulação são duas formas distintas pelas quais a modelagem de sistemas pode ser realizada. A Teoria das Filas é um método de análise que aborda o assunto por meio de fórmulas matemáticas e a Simulação é uma técnica que utiliza o computador para tentar montar um modelo que represente melhor o sistema estudado. A abordagem matemática de filas iniciou-se em 1908, em Copenhague, na Dinamarca, por meio de Agner Krarup Erlang, considerado o pai da Teoria das Filas. Inicialmente aplicado ao redimensionamento de centrais telefônicas, a partir da segunda guerra mundial a teoria foi aplicada a outros problemas de filas. Na década de 60 surgiram as linguagens de simulação e atualmente podem ser facilmente utilizadas [12].

A simulação envolve a descrição do comportamento de um sistema, bem como a construção de teorias e hipóteses, além da utilização de um modelo que possa prever o comportamento futuro [5]. Através das informações obtidas a partir desse modelo, se possibilita a resolução de problemas sem a necessidade de alterações estruturais.

Atualmente, a modelagem de sistemas tem diversas aplicações nas mais diversas áreas, tais como as linhas de produção, onde possui uma maior quantidade de aplicações, nos transportes ferroviário, rodoviário, marítimo e de elevadores, comunicações, bancos, supermercados, escritórios, sistemas de confiabilidade e processamento de dados, entre outros [12].

Dentre as aplicações no sistema portuário, a Teoria das Filas foi aplicada em um estudo de caso para avaliar as características de atendimento aos navios que atracam em um terminal marítimo para carregamento de minério de ferro e manganês. Foi realizada a simulação da operação de um novo píer na fase de sua implantação. Foram simulados os resultados com as capacidades de operação do porto com navios de pequeno e grande porte [3]. Ficou comprovado que com a inclusão do novo píer, houve uma redução no tamanho médio da fila e no tempo médio da fila, mantendo a eficiência operacional do terminal marítimo para embarque de minério de ferro e manganês, com baixa ociosidade e melhores taxas médias de ocupação.

Utilizou-se a Teoria das Filas no estudo de um posto de combustível iraniano [14]. O modelo proposto foi implementado em um caso real, utilizando uma técnica de simulação para estudar o comportamento do novo sistema e os resultados foram comparados com a situação existente. O layout de todos os postos de gasolina foi projetado de forma que, com exceção dos carros da frente, os carros não podem sair até que os outros carros recebam seus serviços. Esse modelo de filas com estado não padrão aumenta o tamanho da fila e o tempo 
de atendimento. A proposta simulada estabelecia que o sistema permanecesse vazio imediatamente para os carros existentes na fila após cada realização do serviço. O cenário foi proposto com o mesmo número de servidores e a mesma restrição de espaço. Ficou demonstrado, através da técnica de simulação adotada, que o desempenho dos postos de gasolina poderia melhorar significativamente se o modelo proposto fosse adotado.

\section{METODOLOGIA}

Os processos metodológicos utilizados nesse artigo são descritos segundo seu propósito, método, técnicas de coleta e técnicas de análise.

Quanto ao propósito, a pesquisa foi classificada como Proposição de Planos, que tem por finalidade apresentar propostas de projetos visando solucionar os problemas já detectados nas organizações [10]. No presente trabalho, o objetivo da pesquisa foi o desenvolvimento de um modelo de simulação para melhorar o atendimento aos clientes de uma distribuidora de combustíveis baseado na Teoria das Filas.

Em relação ao método, a pesquisa classifica-se como predominantemente qualitativa, pois as informações dos trabalhos analisados possuem caráter exploratóriointerpretativo. Quanto ao delineamento, a pesquisa caracteriza-se como um estudo de caso, como uma estratégia de pesquisa pode ser utilizada de modo exploratório, visando levantar questões e hipóteses para estudos posteriores; de modo descritivo, associando variáveis ou até mesmo de maneira explanatória [10].

No que tange as técnicas de coleta, foram adotadas a observação participante de forma aberta e a consulta a documentos, na forma de relatórios mensais. Através da observação participante aberta o pesquisador pode observar, entrevistar e participar do ambiente estudado e a consulta a documentos é uma fonte utilizada para complementar outros métodos de coletas [10]. Em relação à técnica de análise foi adotada a análise de conteúdo, definida como uma técnica que objetiva contar a frequência de um fenômeno, procurando identificar relações entre os mesmos [10].

\section{ESTUDO DE CASO}

O Terminal de Escoamento de Combustíveis da Refinaria de Petróleo está localizado no estado do Rio Grande do Sul e opera com os seguintes produtos: Aguarrás Mineral, Álcool Anidro, Álcool Hidratado, Biodiesel, Gás Liquefeito de Petróleo (GLP), Gasolina, Gasolina Premium, Naftas, Normal Pentano, Óleo Combustível, Óleo de Processo 35, Óleo diesel, Óleo Diesel Marítimo, Querosene Iluminante e Solvoleo 3. Está localizado dentro da Planta Industrial e dispõe de oleodutos para recebimento e carregamento via modal aquaviário. Também possui Ilhas para carregamento via modais rodoviário e ferroviário, disponibilizando ainda pontos dedicados ao recebimento de produtos rodoferroviário.

Em relação ao carregamento por meio de trens, existem seis Ilhas que podem ser utilizadas tanto para carregar gasolina, como também diesel. O sistema possui ainda quatro pontos de recebimento de etanóis. Atualmente o carregamento através desse modal não está sendo utilizado. Quanto ao modal aquaviário, possui ligação através de dutos a um Terminal Portuário, para o recebimento de petróleo bruto e escoamento de alguns derivados. No que tange ao modal rodoviário, a empresa possui quatro Plataformas de Carregamento. A Plataforma 1 é composta de quatro ilhas de abastecimento, as quais podem carregar Diesel, Gasolina e Etanóis. Óleo Combustível é carregado na Plataforma 2. O carregamento dos Solventes e Gasolinas Especiais é realizado na Plataforma 3, a qual possui seis pontos de abastecimentos para os Solventes e um ponto de carregamento para Gasolina Premium. GLP é carregado na Plataforma 4. Todos os caminhões abastecidos nas Plataformas entram e saem pelo Terminal. 


\subsection{PLATAFORMA 1}

A Plataforma 1 do Terminal de Abastecimento realiza o carregamento dos caminhões de Frotas, os quais são responsáveis por levar os produtos até os postos de combustíveis. Também efetua o carregamento dos caminhões de Transferência, que transportam os produtos para outros Terminais de Abastecimento. É composta por quatro Ilhas de abastecimento, subdivididas da seguinte maneira: A Ilha 1 é responsável pelo carregamento de Gasolina, Diesel S-500 e Diesel S-10, assim como a Ilha 2; na Ilha 3 são carregados Gasolina, Diesel S-500 e Álcool Hidratado; na Ilha 4 é possível carregar Gasolina Pura e Diesel S-500, atendendo exclusivamente os caminhões de Transferência. Cada Ilha possui a capacidade de atendimento de dois caminhões. Todas as Ilhas podem atender tanto aos caminhões de Frotas quanto os de Transferência. A coleta dos dados para posterior análise e simulação foi realizada apenas nas Ilhas 1 , 2 e 3 da Plataforma 1 e, portanto, somente com os caminhões de Frota, devido à maior demanda por parte desses veículos.

Atualmente o Álcool Anidro é adicionado à Gasolina A na proporção de 27\%, sendo comercializado nos postos de serviço como Gasolina C [1]. O mesmo ocorre com o Biodiesel, que é adicionado a todo Diesel comercializado em postos, na proporção de $10 \%$, constituindo o Diesel B. O Diesel S-10 possui menor quantidade de enxofre que o S-500. Já o Álcool Hidratado é comercializado nos postos como Etanol, não possuindo misturas. O carregamento dos caminhões de Transferência é realizado sem a adição das misturas [1].

\subsubsection{SISTEMA DE CARREGAMENTO - PLATAFORMA 1}

O carregamento dos caminhões de Frota é efetuado mediante agendamento prévio realizado pelas distribuidoras com, no mínimo, 60 minutos de antecedência, iniciando a partir das 06 horas e 30 minutos da manhã. O último horário de agendamento é às 17 horas e 30 minutos e cada caminhão possui uma tolerância de 15 minutos a menos e a mais no horário agendado. Cada cliente pode agendar até quatro caminhões por hora para carregar, variando em função da demanda negociada por cada uma. Os caminhões podem aguardar no pátio externo do Terminal até a entrada para acesso à Plataforma. O motorista se apresenta no Drive in para conferência do agendamento e da ordem de carregamento, onde há a descrição do tipo de produto e a respectiva quantidade. Após é liberado para acessar a Plataforma, que possui a capacidade máxima de atendimento de seis caminhões, enquanto quatro a seis caminhões aguardam na fila no pátio interno.

Logo que chega à Plataforma, o motorista informa ao funcionário Conferente o produto que irá carregar para que o mesmo efetue a organização na fila de espera. Ao acessar a Ilha é informado ao atendente, denominado Operador de Enchimento, através da Ordem de Carregamento, o código do produto, que já é colocado no caminhão com o percentual de mistura adequado a capacidade do mesmo. Quando ocorre a conclusão do carregamento, o caminhão se dirige para a Ilha de Amostra, onde é adicionado o aditivo e retirada a amostra para análise laboratorial. Somente os caminhões de gasolina retiram amostra, para ser analisado o teor alcoólico da mesma. Em seguida se direciona ao Drive out, onde efetua a entrega da Ordem de Carregamento assinada pelo Conferente ao atendente, o que sinaliza que a operação foi realizada de forma correta, onde é liberada sua saída. Pode-se observar na Figura 2 o fluxograma de funcionamento da Plataforma 1.

Figura 2 - Fluxograma de Funcionamento da Plataforma 1 


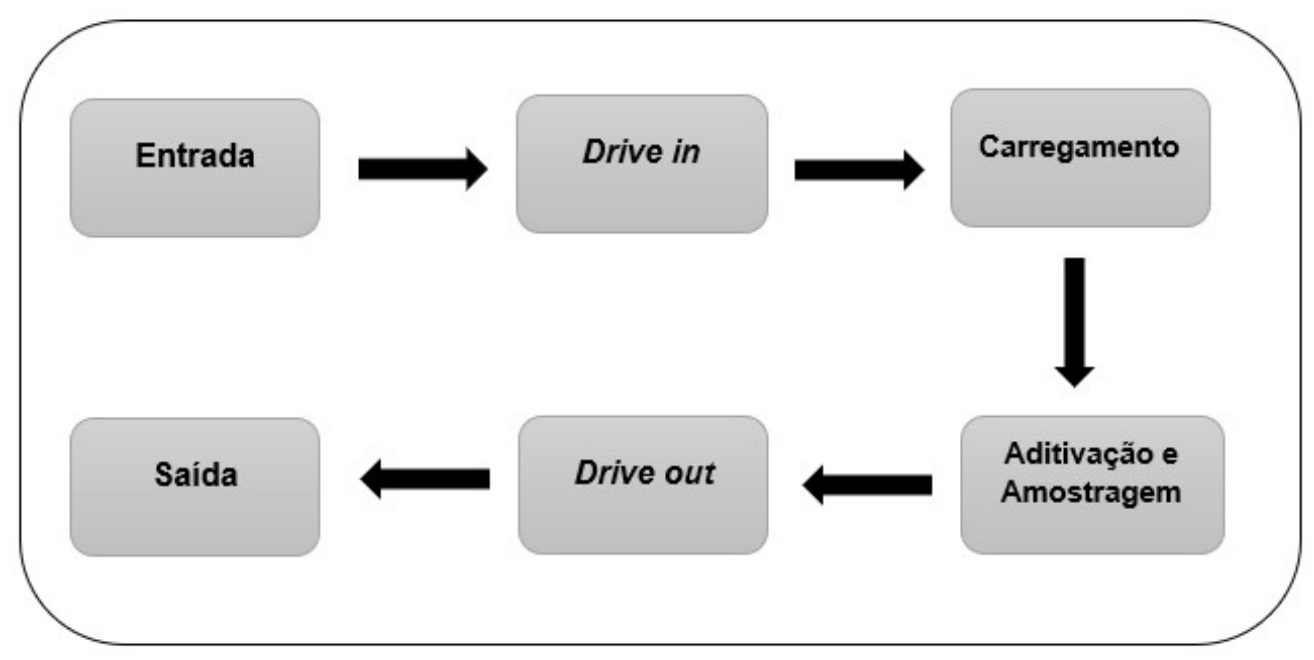

Fonte: Elaborada pelos autores.

\subsection{COLETA E ANÁLISE DE DADOS}

Os dados necessários para a realização da simulação foram obtidos a partir do banco de dados do sistema da empresa, por meio de um relatório de vendas do mês de abril do ano de 2018. A grande vantagem de utilizar estes dados é que, devido o registro acontecer de forma automática, é possível obter uma quantidade expressiva de dados em pouco tempo. Os relatórios incluem os horários de entrada e saída dos caminhões do Terminal, bem a descrição e a quantidade dos produtos carregados, conforme a Tabela 1.

Tabela 1 - Exemplo de relatório similar ao relatório gerado pelo sistema da empresa

\begin{tabular}{ccccccc}
\hline Descrição & Data & Entrada & Saída & Veículo & $\begin{array}{c}\text { Quantidade } \\
\text { Solicitada (L) }\end{array}$ & $\begin{array}{c}\text { Capacidade } \\
\text { Total (L) }\end{array}$ \\
Biodiesel & $02 / 04 / 2018$ & $11: 08$ & $12: 11$ & IQH9115 & 2.200 & \\
Biodiesel & $02 / 04 / 2018$ & $11: 08$ & $12: 11$ & IQH9115 & 2.200 & \\
Óleo Diesel & $02 / 04 / 2018$ & $11: 08$ & $12: 11$ & IQH9115 & 19.800 & \\
Óleo Diesel & $02 / 04 / 2018$ & $11: 08$ & $12: 11$ & IQH9115 & 19.800 & 44.000 \\
Biodiesel & $02 / 04 / 2018$ & $08: 35$ & $08: 59$ & IPK3265 & 500 & \\
Biodiesel & $02 / 04 / 2018$ & $08: 35$ & $08: 59$ & IPK3265 & 300 & \\
Biodiesel & $02 / 04 / 2018$ & $08: 35$ & $08: 59$ & IPK3265 & 200 & \\
Óleo Diesel & $02 / 04 / 2018$ & $08: 35$ & $08: 59$ & IPK3265 & 4.500 & \\
Óleo Diesel & $02 / 04 / 2018$ & $08: 35$ & $08: 59$ & IPK3265 & 2.700 & \\
Óleo Diesel & $02 / 04 / 2018$ & $08: 35$ & $08: 59$ & IPK3265 & 1.800 & \\
Biodiesel & $02 / 04 / 2018$ & $09: 39$ & $10: 58$ & FKD4538 & 500 & \\
Biodiesel & $02 / 04 / 2018$ & $09: 39$ & $10: 58$ & FKD4538 & 500 & \\
Biodiesel & $02 / 04 / 2018$ & $09: 39$ & $10: 58$ & FKD4538 & 500 & \\
Óleo Diesel & $02 / 04 / 2018$ & $09: 39$ & $10: 58$ & FKD4538 & 4.500 & \\
Óleo Diesel & $02 / 04 / 2018$ & $09: 39$ & $10: 58$ & FKD4538 & 4.500 & \\
Óleo Diesel & $02 / 04 / 2018$ & $09: 39$ & $10: 58$ & FKD4538 & 4.500 & \\
\hline \multicolumn{7}{c}{ Fonte: Elaborada pelos autores. } \\
\end{tabular}

Fonte: Elaborada pelos autores.

A amostra analisada foi de 2.353 caminhões, com capacidade de armazenamento entre 2.000 e 65.000 litros. Conforme a Tabela 2 é possível identificar que a capacidade não tem influência no tempo de permanência dos caminhões dentro do Terminal. 
Tabela 2 - Relação dos Tempos de Carregamentos dos Caminhões

\begin{tabular}{ccccc}
\hline Número de Caminhões & Capacidade (L) & Tempo Mínimo & Tempo Médio & Tempo Máximo \\
\hline 360 & $2.000-10.000$ & $00: 19$ & $01: 28$ & $03: 43$ \\
755 & $11.000-20.000$ & $00: 20$ & $01: 24$ & $07: 09$ \\
647 & $21.000-30.000$ & $00: 18$ & $01: 33$ & $06: 06$ \\
174 & $31.000-40.000$ & $00: 46$ & $01: 46$ & $06: 06$ \\
355 & $41.000-50.000$ & $00: 34$ & $01: 32$ & $05: 35$ \\
62 & $60.000-70.000$ & $00: 45$ & $01: 35$ & $02: 53$ \\
\hline
\end{tabular}

Fonte: Elaborada pelos autores.

\subsection{MODELO DE SIMULAÇÃO}

O modelo de simulação foi baseado na Teoria das Filas. Como elemento de uma fila tem-se a população, de onde surgem os clientes que formam a fila e aguardam por algum tipo de serviço [12]. O atendimento se constitui de um ou mais servidores, também chamados de atendentes ou canais de serviço. A disciplina na fila é a regra que define qual deverá ser o próximo a ser atendido, sendo mais comum o "primeiro a chegar é o primeiro a ser atendido" (FIFO: First In First Out). Também podem existir outras disciplinas como o "último a chegar e primeiro a ser atendido" (LIFO: Last In First Out), atendimento por ordem de prioridade, serviço randômico, dentre outros. Em relação ao Tipo da Fila, quando se necessita dimensionar um sistema, podem-se escolher entre diversas opções de atendimento, tais como: única fila e único servidor, única fila e diversos servidores, diversas filas e diversos servidores, filas especiais ou alteração dinâmica no sistema de atendimento.

No que diz respeito às filas, para fins de cálculos, são utilizadas as variáveis randômicas. Dessa forma, para as principais variáveis existe um valor médio e uma distribuição de probabilidades, demonstrando chances de ocorrências dos valores. Na Tabela 3, estão listadas as Variáveis Randômicas Fundamentais.

\begin{tabular}{cc} 
& Tabela 3 - Variáveis Randômicas Fundamentais \\
\hline I & Variáveis referentes ao processo de chegada \\
IC & Taxa Média de Chegada ou Ritmo Médio de Chegada \\
& Intervalo Médio entre Chegadas \\
TF & Variáveis referentes à fila \\
NF & Número Médio de Clientes na Fila \\
& Variáveis referentes ao processo de atendimento \\
TA & Tempo Médio de Atendimento ou de Serviço \\
M & Quantidade de Atendentes \\
NA & Número Médio de Clientes que estão sendo atendidos \\
$\mu$ & Ritmo Médio de Atendimento de cada Servidor \\
& Variáveis referentes ao sistema \\
TS & Tempo Médio de Permanência no Sistema \\
TFS & Tempo Fora do Sistema \\
NS & Número Médio de Transações ou Clientes no Sistema \\
\hline & Fonte: Adaptado [12].
\end{tabular}

Pode-se observar, na Tabela 4, as fórmulas utilizadas para a realização dos cálculos das Variáveis Randômicas Fundamentais. 
Tabela 4 - Fórmulas para calcular as Variáveis Randômicas Fundamentais

\begin{tabular}{lc}
\hline \multicolumn{1}{c}{ Variáveis } & Fórmula \\
Intervalo Médio Entre Chegadas & $\mathrm{IC}=1 / \lambda$ \\
Tempo do Atendimento & $\mathrm{TA}=1 / \mu$ \\
Taxa de Utilização dos Atendentes & $\rho=\lambda / \mathrm{C} . \mu$ \\
Intensidade de Tráfego & $\mathrm{I}=|\lambda / \mu|=|\mathrm{TA} / \mathrm{IC}|$ \\
& $\mathrm{NS}=\mathrm{NF}+\mathrm{NA}$ \\
& $\mathrm{NA}=\lambda / \mu$ \\
Relações entre Filas, Sistemas e Atendimento & $\mathrm{NS}=\mathrm{NF}+\lambda / \mu=\mathrm{NF}+\mathrm{TA} / \mathrm{IC}$ \\
& $\mathrm{TS}=\mathrm{TF}+\mathrm{TA}$ \\
$\mathrm{NA}=\rho=\lambda / \mathrm{C} . \mu$ \\
Fórmulas de Little
\end{tabular}

Fonte: Adaptado [12].

Para o presente estudo, no que tange a Disciplina na Fila foi adotado o modelo FIFO: First In First Out. Quanto ao Tipo de Fila foi utilizado o modelo M/M/c. Esse modelo apresenta uma única fila e diversos servidores e tanto a chegada como o atendimento são marcovianos, pois seguem a Distribuição de Poisson ou a Distribuição Exponencial Negativa [12]. Supõem-se ainda que a capacidade de atendimentos dos servidores seja a mesma e para casos de população infinita. A Fig. 3 apresenta a representação do Modelo M/M/c.

Figura 3 - Representação do Modelo de Filas M/M/c

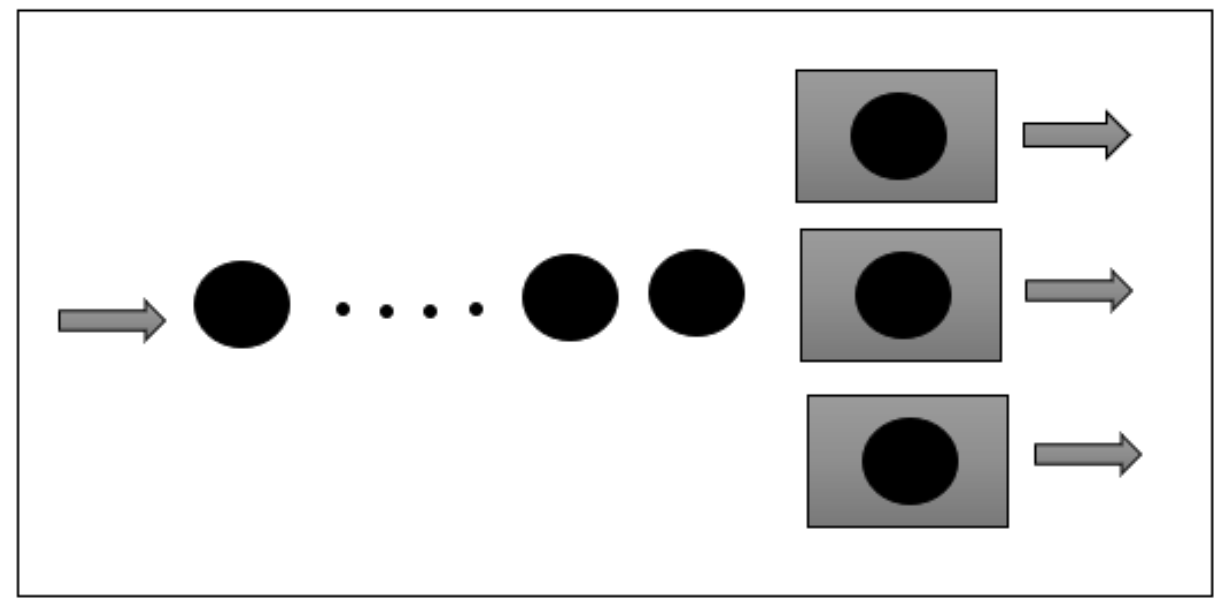

Fonte: Adaptado [12].

Para esse sistema são válidas as seguintes definições: Ritmo Médio de Chegada, Intervalo Médio Entre Chegadas, Tempo Médio de Atendimento ou de Serviço em cada atendente e Ritmo médio de Atendimento de cada atendente [12].

\subsection{DISCUSSÃO DOS DADOS}

A análise dos dados foi realizada, primeiramente, com base nos resultados obtidos na coleta efetuada na empresa objeto de estudo. Para tal, foram efetuados os seguintes 
cálculos: Ritmo Médio de Chegada dos Caminhões, Intervalo Entre Chegadas, Ritmo de Atendimento, Tempo Médio de Atendimento, Taxa de utilização dos Atendente, Número Médio de Clientes no Sistema, Tempo Médio de Permanência no Sistema e o Tempo Médio de Permanência na Fila. Em seguida realiza-se a Simulação através da variação dos dados obtidos.

\subsubsection{RITMO MÉDIO DE CHEGADA}

O ritmo médio de chegada é uma variável que permite quantificar o processo de chegada de dos clientes em uma fila, em um dado intervalo de tempo [12]. Para o cálculo foi utilizado o tamanho da amostra obtida no relatório, que foi de 2.353 caminhões, durante período de 24 dias analisados.

$$
\begin{gathered}
\lambda=\frac{2.353}{24} \\
\lambda=99 \text { caminhões } / \text { dia }
\end{gathered}
$$

Como o tempo de funcionamento do Terminal é de 11 horas/dia, tem-se:

$$
\begin{gathered}
\lambda=\frac{99}{11} \\
\lambda=9 \text { caminhões/hora }
\end{gathered}
$$

Logo, o ritmo médio de chegada à Plataforma é de nove caminhões por hora (Tabela 5).

Tabela 5 - Ritmo médio de chegada à Plataforma.

$\mathrm{N}^{\circ}$ de caminhões Tempo de funcionamento do terminal (horas)

9

1

Fonte: Elaborada pelos autores.

\subsubsection{INTERVALO ENTRE CHEGADAS}

O intervalo entre as chegadas indica o intervalo médio de tempo entre as chegadas dos clientes ao processo de atendimento [12]. É calculado conforme fórmula abaixo:

$$
\begin{array}{r}
\text { IC }=\frac{1}{\lambda} \\
\text { IC }=\frac{1}{9} \\
\text { IC }=0,11 \times 60 \\
\text { IC }=7 \text { minutos }
\end{array}
$$

Desse modo, o intervalo médio de tempo entre as chegadas dos clientes ao processo de atendimento é igual a 7 minutos (Tabela 6).

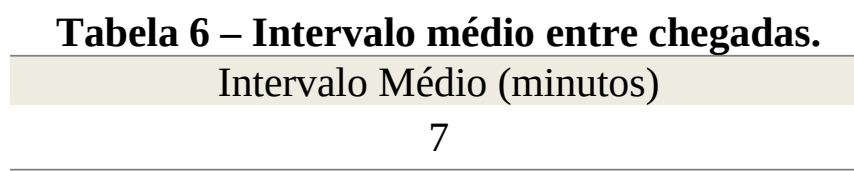

Fonte: Elaborada pelos autores.

\subsubsection{RITMO DE ATENDIMENTO}

Através dessa variável é possível obter a capacidade de atendimento por parte dos atendentes [12]. No caso em estudo, cada uma das três Ilhas de Abastecimento da Plataforma 1 pode atender dois caminhões de cada vez, contando para tal com o número de seis atendentes. 
$\mu=6$ caminhões/hora

Dessa forma, o ritmo de atendimento é de 6 caminhões por hora.

\subsubsection{TEMPO MÉDIO DE ATENDIMENTO}

O tempo médio de atendimento foi definido de acordo com a coleta de dados obtida no relatório. Para isso, foi realizada uma média entre os tempos de atendimento do total de elementos da amostra, conforme a Tabela 7.

Tabela 7 - Tempos de Médio de Atendimentos dos Caminhões

\begin{tabular}{ccccc}
\hline$N^{\circ}$ de caminhões & Capacidade (litros) & Tempo Mínimo & Tempo Médio & Tempo Máximo \\
360 & $2.000-10.000$ & $00: 19$ & $01: 28$ & $03: 43$ \\
755 & $11.000-20.000$ & $00: 20$ & $01: 24$ & $07: 09$ \\
647 & $21.000-30.000$ & $00: 18$ & $01: 33$ & $06: 06$ \\
174 & $31.000-40.000$ & $00: 46$ & $01: 46$ & $06: 06$ \\
355 & $41.000-50.000$ & $00: 34$ & $01: 32$ & $05: 35$ \\
62 & $60.000-70.000$ & $00: 45$ & $01: 35$ & $02: 53$ \\
Total & & $00: 30$ & $01: 33$ & $05: 15$
\end{tabular}

Fonte: Elabora pelos autores.

De acordo com a tabela:

$$
\mathrm{TA}=93 \text { minutos }
$$

Portanto, o tempo médio de atendimento é de 93 minutos.

\subsubsection{NÚMERO MÉDIO DE CLIENTES NO SISTEMA}

O número médio de clientes no sistema dá-se em função da capacidade de atendimento dos atendentes, que é de seis caminhões. Logo,

$$
\mathrm{NS}=6 \text { caminhões }
$$

\subsubsection{TEMPO MÉDIO DE PERMANÊNCIA}

O tempo médio de atendimento informa o período que o caminhão permanece na Ilha de abastecimento, que conforme calculado abaixo, é de 40 minutos.

$$
\begin{gathered}
\text { TS }=\text { NS } / \lambda \\
\text { TS }=6 / 9 \\
\text { TS }=0,67 \times 60 \\
\text { TS }=\mathbf{4 0} \text { minutos }
\end{gathered}
$$

\subsubsection{TEMPO MÉDIO DE PERMANÊNCIA NA FILA}

Essa variável indica o período que os caminhões aguardam na fila de abastecimento, no pátio interno da Plataforma 1, consistindo em um total de 53 minutos.

$$
\begin{gathered}
\mathrm{TS}=\mathrm{TF}+\mathrm{TA} \\
\mathbf{T F}=\mathbf{5 3} \text { minutos }
\end{gathered}
$$

\subsection{SIMULAÇÃO 1}

Para efetuar essa simulação, foi alterado apenas o ritmo médio de chegada, de nove para 12 caminhões por hora.

$$
\lambda=12 \text { caminhões/hora }
$$

Dessa forma, o intervalo entre as chegadas diminuiu:

$$
\text { IC }=\frac{1}{\lambda}
$$




$$
\begin{gathered}
\text { IC }=\frac{1}{12} \\
\text { IC }=0,083 \times 60 \\
\text { IC }=\mathbf{5} \text { minutos }
\end{gathered}
$$

Porém, conforme calculado abaixo, mesmo com a redução no tempo de atendimento em dez minutos, o tamanho da fila aumentou de 53 para 63 minutos.

$$
\begin{gathered}
\mathrm{TA}=93 \text { minutos } \\
\mathrm{TS}=\mathrm{NS} / \lambda \\
\mathrm{TS}=6 / 12 \\
\mathrm{TS}=0,5 \times 60 \\
\mathrm{TS}=\mathbf{3 0} \text { minutos } \\
\mathrm{TS}=\mathrm{TF}+\mathrm{TA} \\
\mathrm{TF}=\mathbf{6 3} \text { minutos }
\end{gathered}
$$

\subsection{SIMULAÇÃO 2}

Para efetuar essa simulação, foi alterado o ritmo médio de atendimento, bem como o número médio de caminhões no sistema, de seis para oito caminhões por hora, porém mantendo o ritmo médio de chegada de 12 caminhões por hora.

$$
\begin{gathered}
\lambda=12 \text { caminhões } / \text { hora } \\
\text { NS }=8 \text { caminhões } \\
\mu=8 \text { caminhões } / \text { hora }
\end{gathered}
$$

Conforme demonstrado abaixo, o tempo de atendimento, bem como o tamanho da fila permaneceram inalterados em relação aos dados reais.

$$
\begin{gathered}
\mathrm{TA}=93 \text { minutos } \\
\mathrm{TS}=\mathrm{NS} / \lambda \\
\mathrm{TS}=8 / 12 \\
\mathrm{TS}=0,666 \times 60 \\
\mathrm{TS}=\mathbf{4 0} \text { minutos } \\
\mathrm{TS}=\mathrm{TF}+\mathrm{TA} \\
\mathbf{T F}=\mathbf{5 3} \text { minutos }
\end{gathered}
$$

\subsection{SIMULAÇÃO 3}

Para efetuar essa simulação, foi alterado o ritmo médio de atendimento, de seis para sete caminhões por hora, mantendo-se o ritmo médio de chegada de nove caminhões.

$$
\begin{gathered}
\lambda=9 \text { caminhões/hora } \\
\text { NS }=7 \text { caminhões } \\
\mu=7 \text { caminhões/hora }
\end{gathered}
$$

Conforme demonstrado abaixo, o tempo de atendimento aumentou para 47 minutos, porém o tamanho da fila reduziu para 36 minutos.

$\mathrm{TA}=83$ minutos

$$
\begin{gathered}
\text { TS }=N S / \lambda \\
\text { TS }=7 / 9 \\
\text { TS }=0,777 \times 60
\end{gathered}
$$




$$
\begin{gathered}
\text { TS }=\mathbf{4 7} \text { minutos } \\
\text { TS }=\text { TF }+ \text { TA } \\
\text { TF }=\mathbf{3 6} \text { minutos }
\end{gathered}
$$

\subsection{SIMULAÇÃO 4}

Para efetuar essa simulação, foi alterado apenas o tempo médio de atendimento, reduzindo-se o mesmo em dez minutos.

$$
\begin{gathered}
\lambda=9 \text { caminhões/hora } \\
\text { NS }=6 \text { caminhões } \\
\mu=6 \text { caminhões } / \text { hora }
\end{gathered}
$$

Conforme demonstrado abaixo, o tempo de permanência no sistema se manteve, porém há uma redução no tempo de permanência na fila.

$$
\begin{gathered}
\mathrm{TA}=83 \text { minutos } \\
\mathrm{TS}=\mathrm{NS} / \lambda \\
\mathrm{TS}=6 / 9 \\
\mathrm{TS}=0,67 \times 60 \\
\mathbf{T}=\mathbf{4 0} \text { minutos } \\
\mathrm{TS}=\mathrm{TF}+\mathrm{TA} \\
\mathbf{T F}=\mathbf{4 3} \text { minutos }
\end{gathered}
$$

\subsection{SIMULAÇÃO 5}

Para efetuar essa simulação, foram alterados o número mensal de caminhões atendidos pela empresa, o período de atendimento e o número de caminhões no sistema. Foi mantido o tempo médio de atendimento da simulação anterior.

$$
\begin{gathered}
\frac{2.800}{24}=116,6=117 \text { caminhões } / \text { dia } \\
\lambda=\frac{117}{12}=9,75 \\
\lambda=\mathbf{1 0} \text { caminhões } / \text { hora } \\
\text { TA }=83 \text { minutos } \\
\text { NS }=7 \text { caminhões } \\
\mu=7 \text { caminhões } / \text { hora }
\end{gathered}
$$

Conforme demonstrado abaixo, o tempo de permanência no sistema aumentou em relação aos dados originais, porém há uma redução no tempo de permanência na fila em 12 minutos.

$$
\begin{gathered}
\mathrm{TS}=\mathrm{NS} / \lambda \\
\mathrm{TS}=7 / 10 \\
\mathrm{TS}=0,7 \times 60 \\
\mathbf{T S}=\mathbf{4 2} \text { minutos } \\
\mathrm{TS}=\mathrm{TF}+\mathrm{TA} \\
\mathbf{T F}=\mathbf{4 1} \text { minutos }
\end{gathered}
$$




\subsection{SIMULAÇÃO 6}

Para efetuar essa simulação, foi alterado apenas o tempo médio de atendimento em relação aos dados originais.

$$
\begin{gathered}
\lambda=9 \text { caminhões/hora } \\
\text { IC }=\frac{1}{\lambda} \\
\text { IC }=\frac{1}{9} \\
\text { IC }=0,11 \times 60=6,6 \\
\text { IC }=7 \text { minutos } \\
\mu=6 \text { caminhões/hora }
\end{gathered}
$$

Conforme demonstrado abaixo, o tempo de permanência no sistema se manteve, porém houve uma redução no tempo de permanência na fila.

$$
\begin{gathered}
\mathrm{TA}=83 \text { minutos } \\
\mathrm{NS}=6 \text { caminhões }
\end{gathered}
$$

$$
\begin{gathered}
\text { TS }=\text { NS } / \lambda \\
\text { TS }=6 / 9 \\
\text { TS }=0,67 \times 60 \\
\text { TS }=\mathbf{4 0} \text { minutos } \\
\text { TS }=\text { TF }+ \text { TA } \\
\text { TF }=\mathbf{4 3} \text { minutos }
\end{gathered}
$$

\begin{tabular}{|c|c|c|c|c|c|c|c|c|}
\hline \multirow[b]{2}{*}{ Variáveis } & \multirow{2}{*}{$\begin{array}{c}\text { Dados } \\
\text { Originais }\end{array}$} & \multicolumn{7}{|c|}{ Simulações } \\
\hline & & 1 & 2 & 3 & 4 & 5 & 6 & 7 \\
\hline $\begin{array}{l}\text { Ritmo de } \\
\text { (caminhões/hora) }\end{array}$ & 9 & 12 & 12 & 9 & 9 & 10 & 9 & 9 \\
\hline $\begin{array}{l}\text { Tempo de Atendimento } \\
\text { (minutos) }\end{array}$ & 93 & 93 & 93 & 83 & 83 & 83 & 83 & 40 \\
\hline $\begin{array}{l}\text { Número de Clientes no } \\
\text { Sistema (caminhões) }\end{array}$ & 6 & 6 & 8 & 7 & 6 & 7 & 6 & 6 \\
\hline $\begin{array}{l}\text { Tempo de Permanência no } \\
\text { Sistema (minutos) }\end{array}$ & 40 & 30 & 40 & 47 & 40 & 42 & 40 & 40 \\
\hline Tempo de Permanência na & 53 & 63 & 53 & 36 & 43 & 41 & 43 & 0 \\
\hline
\end{tabular}

\subsection{SIMULAÇÃO 7}

Para efetuar essa simulação, foi alterado apenas o tempo médio de atendimento em relação aos dados originais, igualando-o ao tempo médio de permanência no sistema.

$$
\begin{aligned}
& \mathrm{TA}=40 \text { minutos } \\
& \mathrm{TS}=40 \text { minutos }
\end{aligned}
$$

Conforme demonstrado abaixo, nessa situação não há filas no sistema.

$$
\begin{gathered}
\mathrm{TS}=\mathrm{TF}+\mathrm{TA} \\
\mathbf{T F}=\mathbf{0} \text { minutos }
\end{gathered}
$$

A Tabela 8 apresenta os principais resultados das simulações.

Tabela 8 - Síntese dos resultados das Simulações 


\section{CONCLUSÕES}

Na simulação 1 foi alterado o ritmo médio de chegada dos caminhões, reduzindo o intervalo de tempo entre as chegadas. Além disso, houve redução no tempo de atendimento, mas aumento no tamanho da fila devido ao maior fluxo de caminhões.

Para efetuar a simulação 2, foram alterados o ritmo médio de atendimento, bem como o número médio de caminhões no sistema e o ritmo médio de chegada. Conforme demonstrado, o tempo de atendimento, bem como o tamanho da fila permaneceram inalterados em relação a situação atual da empresa.

Em relação a simulação 3, foi alterado apenas o ritmo médio de atendimento dos caminhões. Conforme demonstrado abaixo, o tempo de atendimento aumentou. O tamanho da fila reduziu significativamente.

Com a alteração de apenas o tempo médio de atendimento, conforme a simulação 4, o tempo de permanência no sistema se manteve, ocorrendo também uma redução no tempo de permanência na fila.

Na simulação 5, foram alterados o número mensal de caminhões atendidos pela empresa, o período de atendimento, o número de caminhões no atendidos no sistema e o tempo médio de atendimento. Conforme calculado, o tempo de permanência no sistema aumentou em relação a situação atual, porém há uma redução no tempo de permanência na fila em 12 minutos.

Apenas o tempo médio de atendimento foi alterado na simulação 6, ocasionado uma redução no tempo de permanência na fila e mantendo o tempo de permanência no sistema.

Através da simulação 7 foi possível observar um sistema sem filas, onde o tempo de permanência e o tempo médio de atendimento.

À vista disso, ao realizar a análise das 7 simulações realizadas, notou-se que a simulação 7 obteve maior excelência, onde o tempo de fila foi igual a 0 minutos, ou seja, o sistema não apresentaria filas. Contudo, para tal resultado, o tempo de atendimento teria que ser reduzido de 93 minutos para 40 minutos, uma redução de 53 minutos no atendimento. Desse modo, uma solução para a redução do tempo de atendimento seria a implementação de novas ilhas de abastecimento na Plataforma 1, bem como admitir operadores de enchimento, os quais são responsáveis pelo controle do percentual de mistura adequado a capacidade de cada caminhão no processo de abastecimento.

\section{CONSIDERAÇÕES FINAIS}

Este estudo teve como objetivo o desenvolvimento de um modelo de simulação para melhorar o atendimento aos clientes de uma distribuidora de combustíveis baseado na Teoria das Filas. Para tal, foram utilizados como base os dados obtidos através do relatório de vendas da empresa, do mês de abril de 2018. Em relação ao método, a pesquisa classifica-se como predominantemente qualitativa, pois as informações dos trabalhos analisados possuem caráter exploratório-interpretativo e quanto ao delineamento, pode-se caracterizá-la como um estudo de caso.

Os resultados foram obtidos através das simulações, onde se efetuaram alterações nas variáveis como forma de realizar a meta proposta. Na simulação 1 foi possível observar uma redução no tempo de atendimento e um aumento no tamanho da fila devido ao maior fluxo de caminhões. Na simulação 3 constatou-se a redução significativa do tamanho da fila. Em relação à simulação 5, ainda que ocorra um aumento significativo da demanda mensal, se o período de atendimento sofrer uma pequena elevação, há uma redução no tempo de 
permanência dos caminhões na fila.

Como limitação de pesquisa destaca-se a dificuldade em obter os tempos de cada etapa do processo de carregamento dos caminhões, visto que o relatório apresentado informa somente os horários de entrada e saída dos mesmos do Terminal. Foi possível identificar, como lacuna de pesquisa, a restrição dos modais de transporte utilizados no processo, uma vez que na empresa analisada é adotado apenas o transporte rodoviário. Por fim, o tema de pesquisa analisado no presente estudo, pode ser desenvolvido em pesquisas futuras, buscando abranger um número maior de modais de transporte para melhorar o sistema de atendimento aos clientes.

\section{REFERÊNCIAS BIBLIOGRÁFICAS}

[1] ANP - Agência Nacional de Petróleo, Gás Natural e Biocombustíveis. Abastecimento em números. Boletim gerencial. Informações sobre a comercialização de combustíveis, 2019. Disponível em: < http://www.anp.gov.br/wwwanp/>. Acesso em: 23 abr. 2019.

[2] CAMPOS, M.; LOON, W. Petróleo e Abertura. Jornal do Brasil, abr. 2002.

[3] CAMELO, G. R.; COELHO, A. S.; BORGES, R. M.; SOUZA, R. M. de. Teoria das Filas e da Simulação Aplicada ao Embarque de Minério de Ferro e Manganês no Terminal Marítimo de Ponta da Madeira. Cadernos do IME - Série Estatística. Universidade do Estado do Rio de Janeiro - UERJ, v. 29, n. 2, p. 1-16. 2010.

[4] FITZSIMMONS, A. J.; FITZSIMMONS, J. M. Administração de Serviços. 2. ed. Porto Alegre: Bookman, 2000.

[5] FREITAS FILHO, P. Introdução à modelagem e simulação de sistemas com aplicações em Arena. 2. ed. Florianópolis: Visual Books, 2008.

[6] HUSSAIN, R.; ASSAVAPOKEE, T.; KHUMAWALA, T. Supply Chain Management in the Petroleum Industry: Challenges and Opportunities. International Journal of Global Logistics \& Supply Chain Management, v. 1, n. 2, p. 90-97, out. 2006.

[7] IBP - Instituto Brasileiro de Petróleo, Gás e Biocombustíveis. Abastecimento, Petroquímica e Biocombustíveis, 2019. Disponível em: <https://www.ibp.org.br/como-atuamos/abastecimento-pq-bio/>. Acesso em: 25 abr. 2019.

[8] ILOS - Especialistas em Logística e Supply Chain. Trasporte de Combustíveis no Brasil: Investimentos para o abastecimento até 2020, 2013. Disponível em: $<$ http://www.ilos.com.br/web/transporte-de-combustiveis-no-brasil-investimentospara-o-abastecimento-ate-2020/>. Acesso em: 23 abr. 2019.

[9] ILOS - Especialistas em Logística e Supply Chain. Transporte Dutoviário de Combustíveis no Brasil: Desafios e Oportunidades, 2014. Disponível em: $<$ http://www.ilos.com.br/web/transporte-dutoviario-de-combustiveis-no-brasildesafios-e-oportunidade/>. Acesso em: 25 abr. 2019.

[10] ROESCH, S. M. A. Projetos de estágio e de pesquisa em administração. São Paulo: Atlas, 2010.

[11] PINHEIRO, V. A. Simulação do Processo de Carregamento em uma Base Distribuidora de Combustível. 2017. Dissertação (Mestrado em Engenharia Industrial) - Pontifícia Universidade Católica do Rio de Janeiro, Rio de Janeiro. 2017. 
[12] PRADO, D. Teoria das Filas e da Simulação. Belo Horizonte: Editora de Desenvolvimento Gerencial, 1999.

[13] SOARES, A. C. Diagnóstico e modelagem da rede de distribuição de derivados de petróleo no Brasil. 2003. 156 f. Dissertação (Mestrado em Engenharia Industrial) Pontifícia Universidade Católica do Rio de Janeiro, Rio de Janeiro, 2003.

[14] TEIMOURY, E. Modelling and improvement of non-standard queuing systems: a gas station case study. International Journal Applied Decision Sciences, v. 4, n. 4. 2011. 